\title{
Medium-chain carboxylic acids production using consortia from winery wastewater, ruminal fluid and granular sludge
}

\author{
Sharon B. Villegas-Rodríguez ${ }^{*}$, , Germán Buitrón ${ }^{a}$ \\ * presenter, svillegasr@iingen.unam.mx \\ a Laboratory for Research on Advanced Processes for Water Treatment, \\ Unidad Académica Juriquilla, Instituto de Ingeniería, Universidad Nacional \\ Autónoma de México, Mexico
}

\section{HIGHLIGHTS:}

- The highest MCFA production was obtained with an endogenous consortium from winery wastewaters

- Caprylic acid was only produced with ruminal consortia

- Clostridium were enriched in all the cultures (up to $85 \%$ )

BACKGROUND: Chain elongation is an anaerobic fermentation process that produces medium-chain fatty acids (MCFA) -such as caproic acid- from volatile fatty acids (VFA) and ethanol. MCFA have a high added value of up to 10 times more than ethanol (Cavalcante et al., 2017) and up to 5 times more than methane (Kleerebezem et al., 2015). The inoculum source is significant when a robust consortium needs to be obtained for the MCFA production. Adequate syntrophic interactions could increase the productivity of the MCFA and offer economic viability to the process. Clostridium kluyveri (an anaerobic bacterium that is present in some biological wastes), is currently the most widely used microorganism for obtaining MCFA due to its ability to use ethanol and VFA for its metabolism (Bornstein and Barker, 1947; Thauer et al., 1967). This work evaluated the potential of an endogenous consortium from winery wastewaters to produce MCFA (white and red wine manufactured at Querétaro, Mexico). The process performances were compared to other inocula, one harvested from a ruminal fluid (sheep slaughterhouse) and granular anaerobic sludge (flour wastewater treatment). The native winery wastewaters consortium has been exposed to high ethanol concentrations (100 g/L, Vital-Jácome et al., 2020). That could favor not only a faster MCFA production process but also the production of acids with longer carbon chains where higher concentrations of ethanol are required.

RESULTS \& DISCUSSION: The experiments were performed in serum bottles incubated at $37^{\circ} \mathrm{C}$ and $150 \mathrm{rpm}$. Synthetic medium rich in phosphates with an ethanol-acetate ratio of 10:1 (500:50 mmol) was used as a substrate. The bottles were inoculated with $2 \mathrm{~g} \mathrm{TS} \mathrm{L}^{-1}$ for each inoculum at $\mathrm{pH}$ 5.5. Figure 1 shows that the highest production of caproic acid was $5.8 \mathrm{~g} / \mathrm{L}$ using an endogenous winery wastewater microbiota. However, when the ruminal fluid was used as inoculum, Caprylic acid $(2.8 \mathrm{~g} / \mathrm{L})$ was 
produced in addition to caproate $(3.5 \mathrm{~g} / \mathrm{L})$ and heptanoic acid $(2.1 \mathrm{~g} / \mathrm{L})$. Caprylic acid is a medium-chain carboxylic acid with higher added value compared to caproic acid. The use of granular sludge reveals the production of only caproic acid $(3.7 \mathrm{~g} / \mathrm{L})$. Although with all the inoculums caproic acid was obtained, faster production rates were observed with the endogenous consortia of winery wastewaters. That can be explained because the microorganisms were already adapted to elevated ethanol concentrations. The other two inocula required more time to adapt to ethanol $(500 \mathrm{mmol})$. Microbial community analyses indicated that the operational taxonomic unit (OTU) associated with Clostridia (85\%) and Bacteroides were dominant and positively correlated with elevated MCFA productivities. Results also suggested that the microbiome evolved in such a way that the MCFA production was improved.

CONCLUSION: It was evidenced that the highest MCFA production was obtained with an endogenous consortium from winery wastewaters. Higher productivity of caproic acid was observed compared to the other inocula used in this work. Nevertheless, caprylic acid was produced with ruminal fluid. The microbial community analyses indicated that OTUs for Bacteroides spp. and Clostridium spp. were positively correlated with the MCCA production.
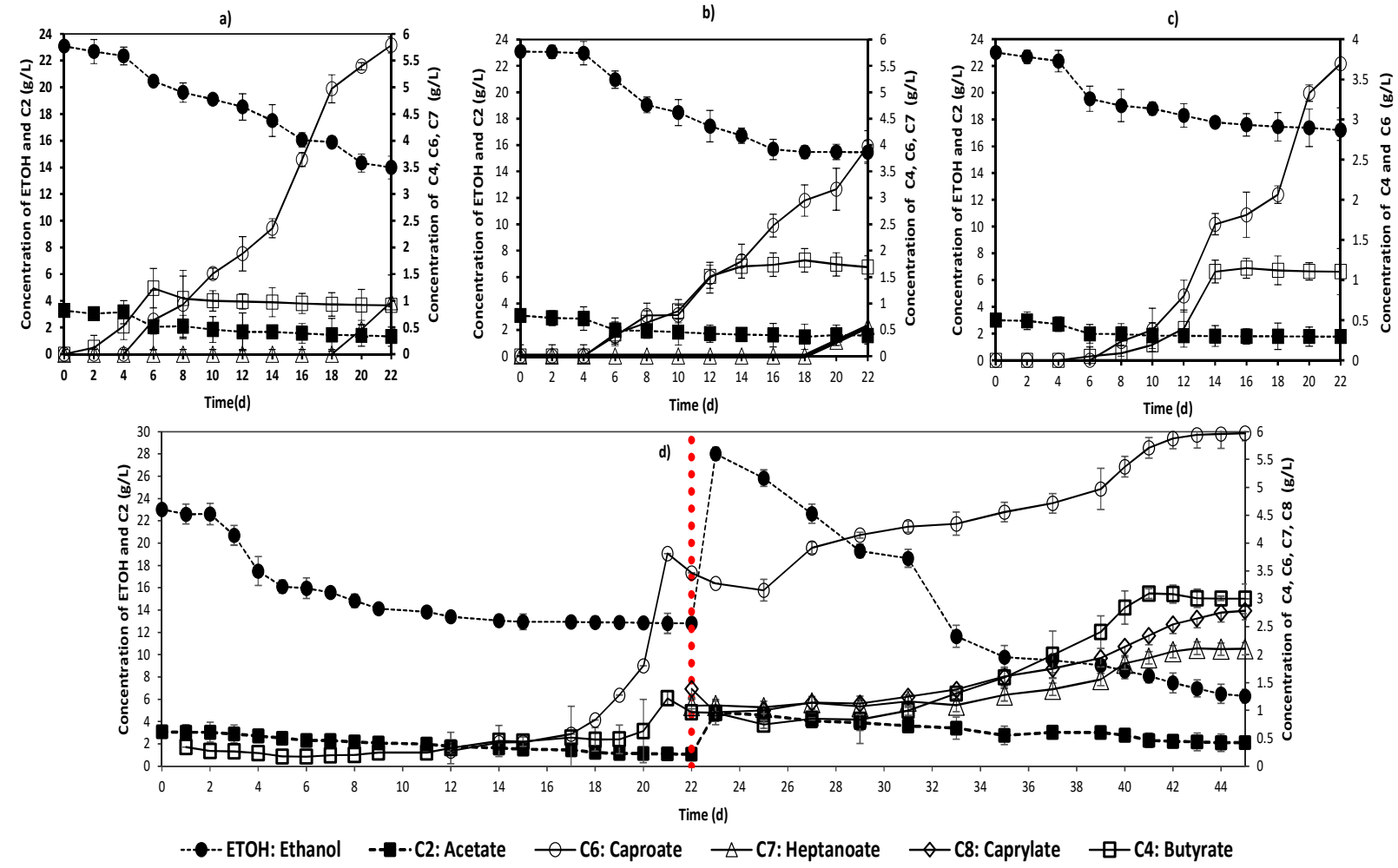

Figure 1. Substrates and fermentation products formation as a function of time using winery wastewaters microbiota (a) red wine; (b) white wine; (c) granular sludge; (d) ruminal fluid. 


\section{ACKNOWLEDGEMENTS}

This wok was supported by the DGAPA-UNAM trough the project PAPIIT IN105119.

\section{REFERENCES}

1. Bornstein, B.T., Barker, H.A., 1947. The energy metabolism of clostridium kluyveri and the synthesis of fatty acids. J. Biol. Chem. 1948 172, 659-669.

2. Cavalcante, W. de A., Leitão, R.C., Gehring, T.A., Angenent, L.T., Santaella, S.T., 2017. Anaerobic fermentation for $n$-caproic acid production: A review. Process Biochem. 54, 106-119. https://doi.org/10.1016/j.procbio.2016.12.024

3. Kleerebezem, R., Joosse. B., Rozendal, R., Loosdrecht, M.C.M., 2015. Anaerobic digestion without biogas? Rev. Environ. Sci. Bio/Technology. https://doi.org/10.1007/s11157-015-9374-6

4. Thauer, R.K., Jungermann, K., Henninger, H., Wenning, J., Decker, K., 1968. The Energy Metabolism of Clostridium kluyveri. Eur. J. Biochem. 4, 173-180. https://doi.org/10.1111/j.1432-1033.1968.tb00189.x

5. Vital-Jacome, M. Cazares-Granillo, M. Carrillo-Reyes, J. and Buitron, G. (2020) Characterization and anaerobic digestion of Mexican highly concentrated wine by-products and effluents, Water Sci Technol, (in press), https://doi.org/10.2166/wst.2020.102 